\title{
Synthesis, Characterizationand Antibacterial Activity of Quaternary Ammonium Compounds Bearing Mixed $N$-Substituted Groups
}

\author{
SOFIANE DAOUDI $^{1}$, ADIL A. OTHMAN ${ }^{1 *}$, TAHAR BENAISSA $^{2}$ \\ and ZAHRA OULD KADA ${ }^{1}$
}

${ }^{1}$ Laboratory of Bioactive Organic Chemistry, Department of Chemistry, Faculty of Sciences, University of Sciences and Technology of Oran -Mohamed Boudiaf, P.O.Box 1505 El-M'naouer, Oran 31003, Algeria

${ }^{2}$ Chemical Physics Laboratory, University of Moulay Tahar, Saida, 20000 Algeria adelaliothman@gmail.com

Received 10 September 2012 / Accepted 23 April 2013

\begin{abstract}
Sixteen quaternary ammonium bromides [QAB]4(a-d)-7(a-d)bearing mixed $N$-branches were synthesized by treating $n$-butyl 2a, cyclohexyl $\mathbf{2 b}, 2,3,4,6$-tetra- $O$-acetyl $-D$ - glucosyl $\mathbf{2 c}$ and cholesteryl 2d bromides, separately with $n$-butyl amine 3a, benzyl amine 3b, $N, N$-dimethyl amino ethyl-2- methyl acrylate $3 \mathbf{c}$ and $N, N$-diethyl amino ethyl-2- methyl acrylate 3d. $N, N$-Diphenyl amine 3e failed to react with any bromides within the scope of this work. The synthesized bromides and QAB were characterized by FT-IR, ${ }^{1} \mathrm{H}-\mathrm{NMR}$ and ${ }^{13} \mathrm{C}-\mathrm{NMR}$. All compounds were tested for their activity against some gram-positive and gram-negative bacteria and some of them exhibited significant antibacterial activities.
\end{abstract}

Keywords: Alkyl and cycloalkyl bromides, Benzyl bromides, Dialkylaminomethyl acrylate bromides, Quaternary ammonium compounds, Antibacterial activity

\section{Introduction}

Quaternary ammonium salts [QAS] found in very important life compounds such as amino acids, central nervous system of animals and cationic bile salts ${ }^{1}$. Some synthesized bile salts were used as good cholesterol-lowering agents ${ }^{2,3}$ and have also been used as antimicrobials ${ }^{4}$, gall stone dissolution enhancers ${ }^{5}$ and DNA transfection agents ${ }^{6,7}$. Several generations of synthetic quaternary ammonium compounds [QAC] which have been used as antimicrobial agents being recognized in the past seven decades. Variations in the four branches attached to quaternary ammonium nitrogen and multiplicity of $\mathrm{N}^{+}$centers were the major features of the classes of QACs ${ }^{8}$. The $N$-alkyl- $N, N$-dimethyl ammonium methyl ethyl acrylates ${ }^{9-13}$ and $N$-alkyl- $N, N$-diethyl ammonium methyl ethyl acrylates ${ }^{10}$ are the raw material for dental antibacterial agents. Two of the new groups with good antimicrobial activity are the heterocyclic pipyridinium ${ }^{14}$ and glucopyranose $\mathrm{QAS}^{15}$. Other increasing applications of QAC were reported in the literature, such as food, beverages preserving agents ${ }^{16}$, fabric ${ }^{17}, \operatorname{cosmetic}^{18}$, medical ${ }^{19}$, environmental ${ }^{20}$ and electrolytes for liquids batteries ${ }^{21}$ uses. Because of the increasing resistance of microorganisms to 
commonly used disinfectants make the synthesis of new types of microbiocides is very important topic. Very few examples of carbo- and heterocyclic branches such as cyclohexane, glycosyl, cholesterol moieties attached directly to the quaternary nitrogen were mentioned in the literature. In this work we report the synthesis of sixteen QAB belong to four classes of amines ( $n$-butyl, benzyl, $N, N$-dimethyl ethyl-2- methyl acrylate amine, $N, N$-diethyl ethyl-2- methyl acrylate amine)and four classes of alkyl bromides bearing cyclohexyl, glycosyl and cholesteryl groups.

\section{Experimental}

All reactions were monitored by TLC, silica gel $\mathrm{F}_{254}$, made by Merck, Germany. The melting points were measured with a BÜCHI 540 melting point apparatus and are uncorrected. The IR spectra were recorded using $\mathrm{KBr}$ discs in a GENESIS II FTIR spectrophotometer, in units of $\mathrm{cm}^{-1}$. The ${ }^{1} \mathrm{H}$ and ${ }^{13} \mathrm{C}$ NMR spectra were recorded on a Bruker AM $300 \mathrm{MHz}$ spectrometer (University of Oran, Essenia) in $\mathrm{CDCl}_{3}$ and referenced to TMS, chemical shifts in ppm. Microorganisms in this study were supplied by the university hospital of Oran and identified in our laboratory. The Mueller Hinton medium was supplied by (Difco).

\section{General procedure for the synthesis of alkyl bromides $\mathbf{2 a}, \mathbf{2} \mathbf{b}$ and $\mathbf{2 d}$}

The alcohols 1a, 1 b and $\mathbf{1 d}(0.067 \mathrm{~mol}), \mathrm{KBr}(0.33 \mathrm{~mol}), \mathrm{H}_{2} \mathrm{O}(20 \mathrm{~mL}), \mathrm{H}_{2} \mathrm{SO}_{4}(10 \mathrm{~mL})$ were added carefully and the mixture was refluxed at $80{ }^{\circ} \mathrm{C}$ for appropriate time until bromide spots on TLC exhibited no change in intensity [average time $7 \mathrm{~h}$ ]. After cooling to room temperature, water $(25 \mathrm{~mL})$ was added and neutralized with $\mathrm{Na}_{2} \mathrm{CO}_{3}$. Extracted with chloroform for three times and dried over anhydrous $\mathrm{MgSO}_{4}$, filtered and evaporated down to dryness to give the bromides $\mathbf{2 a}, \mathbf{2 b}$ and $\mathbf{2 d}$ in yields: $73 \%, 57 \%$ and $87 \%$ respectively.

\section{Preparation of bromo-2, 3, 4, 6-O-acetyl-D-glucopyranoside $\mathbf{2 c}^{22}$}

D-Glucose (1c ) $4.0 \mathrm{~g}, 0.022 \mathrm{~mol}$ was dissolved in acetic anhydride $(20 \mathrm{~mL})$, acetyl bromide (5 $\mathrm{mL})$ and $\mathrm{HClO}_{4}(0.3 \mathrm{~mL})$ added to it and the mixture was left aside for $48 \mathrm{~h}$ at room temperature. The reaction mixture was neutralized with aqueous $\mathrm{Na}_{2} \mathrm{CO}_{3}(25 \mathrm{~mL})$ and extracted with chloroform, dried over anhydrous $\mathrm{MgSO}_{4}$, filtered and evaporated to dryness to give a solid crystalline, recrystallized from diethyl ether to a colorless crystalline $2 \mathrm{c}(7.5 \mathrm{~g}), 85 \%$, m.p. $82^{\circ} \mathrm{C}$.

General procedure for the synthesis of quaternary ammonium bromides 4(a-d) - 7(a-d)

Alkyl bromides 2(a-d)(0.0036 mol), alkyl amines 3(a-e) in dichloromethane $(20 \mathrm{~mL})$ were refluxed for appropriate times and temperatures until TLC showed complete conversions to products. The volatiles were removed under vacuum to give solid products which recrystallized from diethyl ether to give colorless crystalline or as syrup 4(a-d)-7(a-d). Results are summarized in Table 1.

Table 1. Reactants, reflux time and melting points for synthesis of QAB 4(a-d)-7(a-d)

\begin{tabular}{|c|c|c|c|c|c|c|c|c|c|c|c|}
\hline QAS & Bromide & Amine & $\begin{array}{c}\text { Reflux } \\
\mathrm{h}\end{array}$ & $\begin{array}{c}\text { m.p. } \\
{ }^{\circ} \mathrm{C}\end{array}$ & $\begin{array}{c}\text { QAS } \\
\%\end{array}$ & QAS & Bromide & Amine & $\underset{\mathrm{h}}{\text { Reflux }}$ & $\begin{array}{c}\text { m.p. } \\
{ }^{\circ} \mathrm{C}\end{array}$ & $\begin{array}{c}\text { QAS } \\
\%\end{array}$ \\
\hline $4 a$ & $2 a$ & $3 a$ & 6 & 240 & 95 & $6 a$ & $2 \mathbf{a}$ & $3 c$ & 36 & 118 & 62 \\
\hline $4 b$ & $2 b$ & $3 \mathbf{a}$ & 8 & & 91 & $6 b$ & $2 b$ & $3 c$ & 56 & & 46 \\
\hline $4 c$ & $2 c$ & $3 a$ & 6 & & 92 & $6 c$ & $2 c$ & $3 c$ & 8 & & 57 \\
\hline 4d & 2d & $3 a$ & 8 & 127 & 94 & 6d & 2d & $3 c$ & 96 & 137 & 56 \\
\hline $5 a$ & $2 a$ & 3b & 8 & 96 & 90 & $7 a$ & $2 a$ & 3d & 8 & 117 & 94 \\
\hline $5 \mathbf{b}$ & $2 b$ & 3b & 8 & 92 & 78 & $7 b$ & $2 b$ & 3d & 8 & 108 & 94 \\
\hline $5 c$ & $2 c$ & $3 \mathbf{b}$ & 6 & & 93 & $7 c$ & $2 c$ & 3d & 8 & & 89 \\
\hline $5 d$ & 2d & $3 b$ & 8 & 122 & 92 & 7d & 2d & 3d & 8 & 148 & 89 \\
\hline
\end{tabular}




\section{Spectral data of QAB}

Group-A

n-Butyl aminobutyl bromide,[n-butyl-ABB] $4 a$

$v\left(\mathrm{~cm}^{-1}\right): 3444.24\left(\mathrm{~N}^{+}-\mathrm{H}\right)^{24}, 2955.96(\mathrm{C}-\mathrm{H}), 1041.37(\mathrm{C}-\mathrm{N}) . \delta(\mathrm{ppm}): 5.60\left(\underline{\mathbf{H}}-\mathrm{N}^{+}\right), 3.32\left(\underline{\mathbf{H}}_{2} \mathrm{C}-\right.$ $\left.\mathrm{N}^{+}\right), 1.98\left(-\underline{\mathrm{CH}}_{2}-\right), 46.94\left(\mathrm{H}_{2} \underline{\mathbf{C}}-\mathrm{N}^{+}\right)^{25}, 26.34\left(-\underline{\mathbf{C H}}_{2^{-}}\right)$.

Cyclohexyl aminobutyl bromide, [cyclohexyl -ABB] $4 \boldsymbol{b}$

$v\left(\mathrm{~cm}^{-1}\right): 3445.21\left(\mathrm{~N}^{+}-\mathrm{H}\right), 2963.09(\mathrm{C}-\mathrm{H}), 1040.41(\mathrm{C}-\mathrm{N}) . \delta(\mathrm{ppm}): 5.53\left(\underline{\mathbf{H}}-\mathrm{N}^{+}\right), 3.30\left(\underline{\mathbf{H}}_{2} \mathrm{C}-\right.$ $\left.\mathrm{N}^{+}\right), 1.94\left(-\underline{\mathrm{C}}_{2}-\right), 1.92(-\mathrm{C} \underline{\mathbf{H}}-\mathrm{cycl}), 47.26\left(\mathrm{H}_{2} \underline{\mathbf{C}}-\mathrm{N}^{+}\right), 37.92\left(-\underline{\mathbf{C H}}_{2}-\right), 36.21$ (-ㅌH-cycl).

2,3,4,6-Tetra-O-acetylglucosyl aminobutyl bromide, [glucosyl-ABB] 4c $v\left(\mathrm{~cm}^{-1}\right):$ : $3284.18\left(\mathrm{~N}^{+}-\mathrm{H}\right), 2931.27(\mathrm{C}-\mathrm{H}), 1736.58(\mathrm{C}=\mathrm{O}), 1244.83(\mathrm{C}-\mathrm{N}) 1031.73(\mathrm{C}-\mathrm{O}), \delta$ $(\mathrm{ppm}): \quad 5.37 \quad\left(\underline{\mathbf{H}}-\mathrm{N}^{+}\right), \quad 3.40\left(\underline{\mathbf{H}}_{2} \mathrm{C}-\mathrm{N}^{+}\right), \quad 2.08\left(-\underline{\mathrm{H}}_{2}\right), \quad 6.40\left(-\underline{\mathrm{C}}_{2} \mathrm{CO}\right), \quad 6.41(\mathrm{C} \underline{\mathrm{HO}}-\mathrm{glu})$, 46.95 $\left(\mathrm{H}_{2} \underline{\mathbf{C}}-\mathrm{N}^{+}\right), 29.54\left(-\underline{\mathbf{C H}}_{2}-\right), 93.21(\underline{\mathbf{C}} \mathrm{HO}-\mathrm{glu})$.

Cholestryl aminobutyl bromide,[cholestryl-ABB] 4d

$v\left(\mathrm{~cm}^{-1}\right): 3419.17\left(\mathrm{~N}^{+}-\mathrm{H}\right), 2935.13(\mathrm{C}-\mathrm{H}), 1633.41(\mathrm{C}=\mathrm{C}), 1373.93(\mathrm{C}-\mathrm{N}), \delta(\mathrm{ppm}): 5.76\left(\underline{\mathbf{H}}-\mathrm{N}^{+}\right)$,


cycl).

\section{Group-B}

n-Butyl aminobenzyl bromides [n-butyl-ABzB] 5 a

$v\left(\mathrm{~cm}^{-1}\right): 3444.17\left(\mathrm{~N}^{+}-\mathrm{H}\right), 3020.73(=\mathrm{C}-\mathrm{H}), 2957.30(\mathrm{C}-\mathrm{H}), 1459.85(\mathrm{C}=\mathrm{C}), 1054.87(\mathrm{C}-\mathrm{N})$, $\delta(\mathrm{ppm}): 5.77\left(\underline{\mathbf{H}}-\mathrm{N}^{+}\right), 3.33\left(-\mathrm{CH}_{2}-\underline{\mathrm{C}}_{2}-\mathrm{N}^{+}\right), 1.94\left(-\underline{\mathrm{C}}_{2}-\mathrm{CH}_{2}-\mathrm{N}^{+}\right), 1.70\left(-\mathrm{C}_{2}-\left(\mathrm{CH}_{2}\right)_{2}-\mathrm{N}^{+}\right), 2.57$ $\left(-\mathrm{N}^{+}-\underline{\mathbf{H}}_{2}-\mathrm{Ph}\right), 7.57$ (프-Ar), 131.95, 128.67, 128.02, 127.19 (뜨-Ar), $55.74\left(-\mathrm{N}^{+}-\underline{C H}_{2}-\mathrm{Ph}\right)$, $46.92\left(\mathrm{~N}^{+}-\underline{\mathrm{CH}_{2}}{ }^{-}\right), 6.35\left(-\left(\underline{\mathrm{CH}_{2}}\right)_{2}-\mathrm{CH}_{2}-\mathrm{N}^{+}\right)$.

Cyclohexyl aminobenzyl bromides [Cyclohexyl-ABzB] $\mathbf{5 b}$

$v:\left(\mathrm{cm}^{-1}\right): 3361.32\left(\mathrm{~N}^{+}-\mathrm{H}\right), 2930.31(\mathrm{C}-\mathrm{H}), 1643.05(\mathrm{C}=\mathrm{C}), 1070.30(\mathrm{C}-\mathrm{N})$.

2,3,4,6-Tetra-O-acetylglucosyl aminobenzyl bromides [glucosyl-ABzB] 5c

$v\left(\mathrm{~cm}^{-1}\right): 3423.04\left(\mathrm{~N}^{+}-\mathrm{H}\right), 2961.16(\mathrm{C}-\mathrm{H}), 1649.80(\mathrm{C}=\mathrm{O}), 1449.24(\mathrm{C}=\mathrm{C}), 1293.04(\mathrm{C}-\mathrm{N})$, $1031.73(\mathrm{C}-\mathrm{O}), \delta(\mathrm{ppm}): 6.46\left(\underline{\mathbf{H}}-\mathrm{N}^{+}\right), 5.78\left(\mathrm{~N}^{+}-\mathrm{C} \underline{\mathbf{H}}-\mathrm{O}-\right), 6.81\left(\mathrm{CH}_{2} \mathrm{CO}\right),, 1.68\left(-\mathrm{N}^{+}-\underline{\mathrm{H}}_{2}-\mathrm{Ph}\right)$,

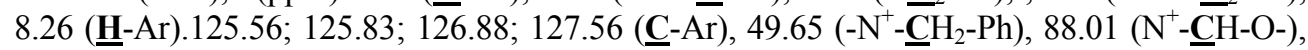
$170.40\left(-\mathrm{CH}_{2} \underline{\mathrm{CO}}\right)$.

Cholestryl aminobenzyl bromides [glucosyl-ABzB] 5d

$v\left(\mathrm{~cm}^{-1}\right): 3400.55\left(\mathrm{~N}^{+}-\mathrm{H}\right), 2935.13(\mathrm{C}-\mathrm{H}), 1644.02(\mathrm{C}=\mathrm{C}), 1050.80(\mathrm{C}-\mathrm{N}), \delta(\mathrm{ppm}): 5.37$


140.79 (C=ㅡ aliphatic), 130.78, 128.30, 128.62, 128.50 (ㄷ-Ar).

\section{Group-C}

n-Butyl amino dimethylaminoethyl-2-methyl acrylate bromides [n-butyl-ABzB] $6 \boldsymbol{a}$ $v\left(\mathrm{~cm}^{-1}\right): 2945.10(\mathrm{C}-\mathrm{H}), 1718.50(\mathrm{C}=\mathrm{O}), 1633.60(\mathrm{C}=\mathrm{C}), 1045.30(\mathrm{C}-\mathrm{N}), 1164.90(\mathrm{C}-\mathrm{O}), \delta$ $(\mathrm{ppm}): 3.67\left(\underline{\mathbf{H}}_{3} \mathrm{C}-\mathrm{N}^{+}\right), 4.07\left(-\underline{\mathbf{H}}_{2} \mathrm{C}-\mathrm{N}^{+}\right), 4.07\left(-\mathrm{C} \underline{\mathbf{H}}_{2} \mathrm{CO}\right), 6.06\left(\underline{\mathrm{CH}}_{2} \mathrm{CO}\right), 4.59\left(\underline{\mathbf{H}}_{2} \mathrm{C}-\right.$ $\left.\mathrm{N}^{+}\right) .62 .21\left(\mathrm{H}_{2} \underline{\mathbf{C}}-\mathrm{N}^{+}\right), 51.79\left(-\mathrm{H}_{2} \underline{\mathbf{C}}-\mathrm{N}^{+}\right), 62.23\left(\mathrm{~N}^{+}-\underline{\mathbf{C}} \mathrm{H}_{2} \mathrm{CH}_{2}-\mathrm{O}\right), 24.69\left(-\underline{\mathbf{C}} \mathrm{H}_{2} \mathrm{CO}\right), 166.30$ $\left(-\mathrm{CH}_{2} \underline{\mathrm{CO}}\right)$. 
Cyclohexyldimethylaminoethyl-2-methyl acrylate bromides[cyclohexyl-DMAEMAB] $6 \mathbf{b}$ $v\left(\mathrm{~cm}^{-1}\right): 2954.05(\mathrm{C}-\mathrm{H}), 1719.23(\mathrm{C}=\mathrm{O}), 1633.48(\mathrm{C}=\mathrm{C}), 1154.39(\mathrm{C}-\mathrm{O}) 1045.23(\mathrm{C}-\mathrm{N}), \delta$

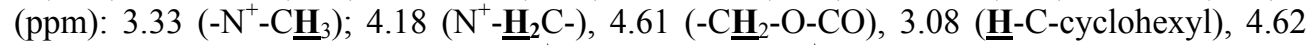
$(\underline{\mathbf{H}}-\mathrm{C}=\mathrm{C}), 1.58\left(\mathrm{C}_{3}-\mathrm{C}=\mathrm{C}\right), 61.26\left(-\mathrm{N}^{+}-\underline{\mathbf{C H}}_{3}\right) ; 53.96\left(-\mathrm{N}^{+}-\underline{\mathbf{C H}}_{2}\right) ; 63.00\left(-\underline{\mathrm{CH}}_{2}-\mathrm{O}-\mathrm{CO}\right) ; 56.56$ (H-C-cyclohexyl); $134.51\left(\mathrm{CH}_{2}-\mathrm{C}=\underline{\mathbf{C H}}_{2}\right)$.

2,3,4,6-Tetra-O-acetylglucosyl dimethylaminoethyl-2-methyl acrylate bromides [glucosyl-DMAEMAB] 6c

$v\left(\mathrm{~cm}^{-1}\right)$ : $2964.05(\mathrm{C}-\mathrm{H}), 1742.37(\mathrm{C}=\mathrm{O}), 1645.05(\mathrm{C}=\mathrm{C}), 1141.65(\mathrm{C}-\mathrm{N}), 1035.55(\mathrm{C}-\mathrm{O}), \delta(\mathrm{ppm})$ :



Cholestryl dimethylaminoethyl-2-methyl acrylate bromides [cholestryl-DMAEMAB] 6d $v\left(\mathrm{~cm}^{-1}\right): 2929.7(\mathrm{CH}), 1728.10(\mathrm{C}=\mathrm{O}), 1602.70(\mathrm{C}=\mathrm{C}), 1126.40(\mathrm{C}-\mathrm{N}), 1066.90(\mathrm{C}-\mathrm{O}), \delta$ (ppm): $3.33\left(-\mathrm{N}^{+}-\underline{\mathbf{H}}_{3}\right) ; 5.34\left(\mathrm{~N}^{+}-\underline{\mathbf{H}}_{2} \mathrm{C}-\right), 4.64$ (-C $\left.\underline{\mathbf{H}}_{2}-\mathrm{O}-\mathrm{CO}\right), 3.41(\underline{\mathbf{H}}-\mathrm{C}-\mathrm{cyclohexyl}), 5.67$ $(\underline{\mathbf{H}}-\mathrm{C}=\mathrm{C}), 76.95\left(-\mathrm{N}^{+}-\underline{\mathbf{C}} \mathrm{H}_{3}\right) ; 59.35\left(\mathrm{~N}^{+}-\mathrm{H}_{2} \underline{\mathbf{C}}-\right), 50.48\left(-\mathrm{CH}_{2} \underline{\mathbf{C}}=\mathrm{O}\right), 135.58\left(\mathrm{CH}_{3}-\underline{\mathbf{C}}=\mathrm{C}\right), 141.12$ $(\underline{-} \underline{\mathrm{CH}}=\mathrm{C}), 166.88\left(\underline{\mathrm{CO}}-\mathrm{CH}_{2}\right)$.

\section{Group D}

n-Butyl amino diethylaminoethyl-2-methyl acrylate bromides [n-butyl-DEAEMAB] 7a: $v\left(\mathrm{~cm}^{-1}\right): 2945.10(\mathrm{C}-\mathrm{H}), 1718.50(\mathrm{C}=\mathrm{O}), 1633.60(\mathrm{C}=\mathrm{C}), 1164.90(\mathrm{C}-\mathrm{O}), \delta(\mathrm{ppm}): 3.57$ $\left(\underline{\mathbf{H}}_{2} \mathrm{C}-\mathrm{N}^{+}\right), 3.87\left(-\mathrm{OC} \underline{\mathbf{H}}_{2}-\mathrm{CH}_{2}-\mathrm{N}^{+}\right), 4.57\left(-\mathrm{OCH}_{2}-\underline{\mathrm{C}}_{2}-\mathrm{N}^{+}\right), 6.04(\underline{\mathbf{H}}-\mathrm{C}=\mathrm{C}) .57 .86\left(\mathrm{H}_{2} \underline{\mathbf{C}}-\mathrm{N}^{+}\right)$, $58.74\left(\mathrm{~N}^{+}-\underline{\mathbf{C}} \mathrm{H}_{2} \mathrm{CH}_{2}-\mathrm{O}\right), 24.02\left(\mathrm{~N}^{+}-\mathbf{C H}_{2} \underline{\mathbf{C}} \mathrm{H}_{2}-\mathrm{O}\right), 166.35\left(-\mathrm{CH}_{2} \underline{\mathbf{C O}}\right), 135.1\left(\mathrm{C}=\underline{\mathbf{C}}-\mathrm{CH}_{2}\right)$.

Cyclohexyldiethylaminoethyl-2-methyl acrylate bromides [cyclohexyl-DEAEMAB] $7 \boldsymbol{b}$ $v\left(\mathrm{~cm}^{-1}\right): 2931.27(\mathrm{C}-\mathrm{H}), 1720.19(\mathrm{C}=\mathrm{O}), 1635.3(\mathrm{C}=\mathrm{C}), 1165.72(\mathrm{C}-\mathrm{O}), 1295(\mathrm{C}-\mathrm{N})$.

2,3,4,6-Tetra-O-acetylglucosyldiethylaminoethyl-2-methyl acrylate bromides [glucosyl$D E A E M A B] 7 c$

$v\left(\mathrm{~cm}^{-1}\right): 2971.77(\mathrm{C}-\mathrm{H}), 1728.22(\mathrm{C}=\mathrm{O}), 1636.30(\mathrm{C}=\mathrm{C}), 1150.94(\mathrm{C}-\mathrm{N}), 1037.62(\mathrm{C}-\mathrm{O}), \delta$ (ppm): $3.33\left(\mathbf{C H}_{3}-\mathrm{CH}_{2}-\mathrm{N}^{+}\right), 3.94\left(\mathbf{H}_{2} \mathrm{C}-\mathrm{N}^{+}\right), 4.53\left(-\mathrm{OCH}_{2}-\underline{\mathrm{H}}_{2}-\mathrm{N}^{+}\right), 4.74\left(\mathrm{~N}^{+}-\mathbf{C H}_{2} \underline{\mathrm{C}}_{2}-\mathrm{O}\right)$, 4.20, 4.06 (ㅍ-C-O-Ac).

Cholestryl diethylaminoethyl-2-methyl acrylate bromides [cholestryl -DEAEMAB] 7d $v\left(\mathrm{~cm}^{-1}\right)$ : $2936.13(\mathrm{C}-\mathrm{H}), 1727.91(\mathrm{C}=\mathrm{O}), 1647.33(\mathrm{C}=\mathrm{C}), 1135.07(\mathrm{C}-\mathrm{N}), 1053.91(\mathrm{C}-\mathrm{O})$.

\section{Antibacterial susceptibility testing}

A disk diffusion assay according to the standard protocols (NCCLS, 2003, 2005; CLSI, 2006) was used ${ }^{26-28}$ in duplicates to determine the susceptibility of three gram-negative bacteria Shigella, Pseudomonas fluorescens and Pseudomonas aeruginosa ATCC 10145 and one gram-positive bacteria Staphylococcus aureus ATCC 25923, using gentamycin and amoxicillinas references .The bacterial suspension (in $0.9 \% \mathrm{NaCl}$ ) turbidity were adjusted to $0.5 \mathrm{Mc}$ Farland, then the suspensions were spread with a sterile cotton swab confluently over the entire surface of Mueller Hinton agar (Merck, Germany).This agar medium was inoculated with $0.5 \mathrm{~mL}$ of cultures containing about $10^{6} \mathrm{CFU} / \mathrm{mL}$. Filter paper disks $(5 \mathrm{~mm}$ diameter) saturated with dimethylsulphoxide (10\% DMSO v:v) solutions of each compound was placed on the indicated agar medium. The incubation time was $24 \mathrm{~h}$ at $37^{\circ} \mathrm{C}$. The blank test disk with DMSO was used. Inhibitory activity was evaluated by measuring the diameter of clear zone observed around the disk in $\mathrm{mm}$ (c.f. Table 5). 
The minimum inhibition concentration (MIC) Tests Microorganisms not affected by compounds tested, no further dilution tests were conducted. Each $1 \mathrm{~mL}$ of the original concentration $\left(10 \mu \mathrm{g} \mathrm{mL}^{-1}\right)$ in DMSO of the compounds $\mathbf{4 ( a - d ) - 7 ( a - d ) ~ w e r e ~ d i l u t e d ~ w i t h ~}$ DMSO in test tubes to $5.0 \mu \mathrm{g} \mathrm{mL}{ }^{-1}$ and optical density at $600 \mathrm{~nm}$ was measured at $24 \mathrm{~h}$.

\section{Results and Discussion}

\section{Synthesis of bromides}

n-Butyl bromide $\mathbf{2 a}$, cyclohexyl bromide $\mathbf{2 b}$, glycosyl bromide $\mathbf{2 c}$ and cholesteryl bromide $\mathbf{2 d}^{23}$ were prepared by refluxing the corresponding alcohols $\mathbf{1 a}, \mathbf{1 b}$ and1d with $\mathrm{KBr}$ in $\mathrm{H}_{2} \mathrm{SO}_{4}$, while 2,3,4,6-tetra-O-acetyl bromide 2c was obtained according to literature method $^{22}$ by treating $D$-glucosewith acetyl bromide in acetic anhydride and catalytic amount of $\mathrm{HClO}_{4}$. The resulted bromides2(a-d) are exhibited in Scheme 1.



Scheme 1, Synthesis of bromides 2(a-d)

All bromides 2(a-d) were isolated and characterized by measuring melting points of solid substances and by spectral analysis of IR as shown in Table 2.

Table 2, Melting points and IR of bromides 2(a-d)

\begin{tabular}{llcc}
\hline Comp & $\mathrm{Mp}^{\circ} \mathrm{C}$ & \multicolumn{2}{c}{$\mathrm{IR} v_{\max }, \mathrm{cm}^{-1}$} \\
\hline & & $\mathrm{OAC}$ & $\mathrm{C}-\mathrm{Br}$ \\
\hline 2a & & & 611 \\
2b & & & 557 \\
2c & 82 & 1737.7 & 605 \\
2d & 151 & & 621 \\
\hline
\end{tabular}

Synthesis of quaternary ammonium bromides[QAB]4(a-d)-7(a-d)

The four bromides $\mathbf{2 ( a - d )}$ were treated with five readily available amines 3(a-e) namely : $n$-butyl amine (3a), benzyl amine (3b), N,N-dimethyl ethyl-2- methyl acrylate amine (3c), $N, N$-diethyl ethyl 2-methyl acrylate amine (3d) and $N, N$-diphenyl amine(3e) revealed only sixteen QAB 4(a-d)-7(a-d).

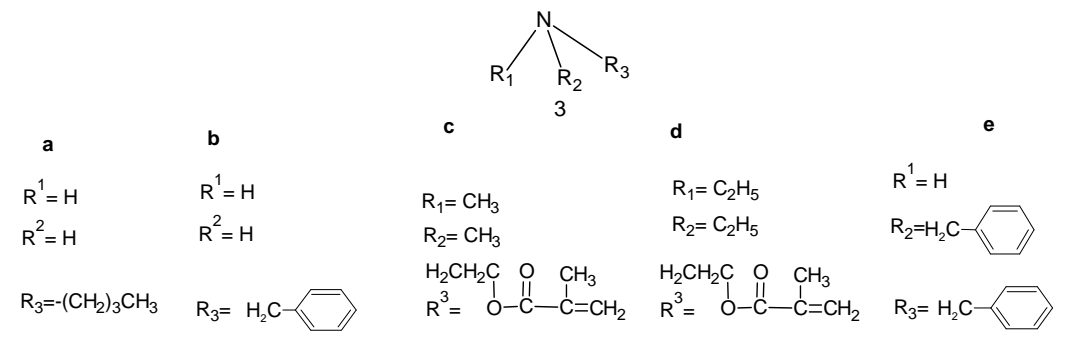

Figure 1. The amines used for the synthesis of $\mathrm{QAB}$ 
$N, N$-Diphenyl amine 3e was failed to react with any of the already synthesized bromides 2(a-d) due to its low nucleophilicity caused by resonance phenomenon between the lone pair of electrons in nitrogen-amine and the two adjacent phenyl groups.

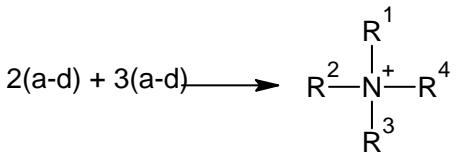

$$
\begin{aligned}
& \text { 4(a-d) - 7(a-d) }
\end{aligned}
$$

Scheme 2. Synthesis of QAB from bromides 2(a-d) and amines 3(a-d)

The sixteen QAB will be categorized into Four Groups named after their amines. Group-A are made of four alkyl aminobutyl bromides [alkyl-ABB]4(a-d), they are: [n-butyl-ABB]4a, [cyclohexyl-ABB]4b, [glucosidyl-ABB]4c and [cholesteryl-ABB]4d. Group-B are comprise of four alkyl aminobenzyl bromides [alkyl-ABzB]5(a-d), they are: [n-butyl-ABzB](5a), [cyclohexyl-ABzB](5b), [glucosidyl-ABzB](5c) and [cholesteryl-ABzB]5d. Group-C are made of four alkyl dimethylaminoethyl-2-methyl acrylate bromides [alkyl-DMAEMAB]6(a-d), they are: [n-butyl-DMAEMAB]6a, [cyclohexyl-DMAEMAB]6b, [glucosidyl-DMAEMAB] $\mathbf{6 c}$ and [cholesteryl-DMAEMAB](6d). Group-D are composed of four alkyl diethylaminoethyl-2-methyl acrylate bromides [alkyl-DEAEMAB]7(a-d), they are: [n-butylDEAEMAB]7a, [cyclohexyl-DEAEMAB]7b, [glucosidyl-DEAEMAB]7c and [cholesterylDEAEMAB]7d. Table 3 shows the compositions, melting points of solid products, IR, ${ }^{1} \mathrm{H}-$ and ${ }^{13} \mathrm{C}-\mathrm{NMR}$ spectroscopic data for some common characteristic groups.

Characterization of $Q A B$ by ${ }^{1} H-$ and ${ }^{13} C-N M R$

There are some selected structural fragments which are common in all QAB groups can be used to detect the formation of the particular QAB such as of Group A as shown in Table 3.

Table 3. Literature data ${ }^{1} \mathrm{H}-,{ }^{13} \mathrm{C}-\mathrm{NMR}^{24}$ in ppm of selected fragments which are common in QAB 4(a-d)-7(a-d).



Note: Data are for the bold letters 


\section{Characterization of Group- $A=[$ alkyl-ABB] $4(a-d)$}

The formations of $n$-butyl ABB were detected by thin layer chromatography (TLC) via their $\mathrm{R}_{\mathrm{f}}$ which were different from starting materials. The IR spectra of 4(a-d) showed bands between 3445-3284 $\mathrm{cm}^{-1}$ for $\mathrm{H}-\mathrm{N}^{+}$bonds, while $4 \mathrm{c}^{24}$ exhibited an additional band at $1736 \mathrm{~cm}^{-1}$ for acetate groups and 4d showed an absorption at $1633 \mathrm{~cm}^{-1}$ indicating the presence of $\mathrm{C}=\mathrm{C}$ for cholesterol. ${ }^{1} \mathrm{H}-\mathrm{NMR}$ spectra showed the following signals between 5.76 to $5.37 \mathrm{ppm}$ for $\mathrm{N}^{+}-\mathbf{H}$, while $\mathrm{N}^{+}-\mathrm{CH}_{2}-$ signals exhibited between 3.30-3.40 ppm. ${ }^{13} \mathrm{C}-\mathrm{NMR}$ spectra for compounds 4(a-d), the groups $\mathrm{N}^{+}-\mathrm{CH}_{2}-$ exhibited signals 46.94, 47.26, 46.95 and 50.17 ppm while the another $-\mathrm{CH}_{2}-$ pieces in $\mathrm{N}^{+}-\mathrm{CH}_{2} \mathrm{CH}_{2}-$ fragments were shown in 26.34, 37.92, 29.54 and 29.50 ppmin 4(a-d) respectively and they are very close to literature values ( Tables 3 and 4$)^{25}$.

\section{Characterization of Group-B = [alkyl-ABzB] 5(a-d)}

The [alkyl-ABzB] 5(a-d) have almost similar groups $\mathrm{N}^{+}-\mathrm{H}, \mathrm{N}^{+}-\mathrm{CH}_{2} \mathrm{CH}_{2}-$ as in Group A, in addition they have the benzyl group $-\mathrm{CH}_{2} \mathrm{Ph}$. IR spectra of of the members of this group showed similar $\mathrm{N}^{+}-\mathrm{H}$ stretching between $3444-3400 \mathrm{~cm}^{-1}$ and additional aromatic $\mathrm{C}=\mathrm{C}$ stretching at the region $1640 \mathrm{~cm}^{-1}$. ${ }^{1} \mathrm{H}-\mathrm{NMR}$ spectra for $\mathrm{N}^{+}-\mathrm{H}$ showed at 6.46-5.77 ppm while $\mathrm{N}^{+}-\mathrm{CH}_{2}-\mathbf{P h}$ signals exhibited between 2.57-1.86 ppm and phenyl protons entered at $8.26,8.16$ and $7.57 \mathrm{ppm} .{ }^{13} \mathrm{C}-\mathrm{NMR}$ spectra for the same fragments are shown in 55.74 $\left(\mathrm{N}^{+}-\mathrm{CH}_{2}-\mathrm{Ph}\right)$ and 131.95-127.19 ppm for aromatic ring carbon. Rest spectral data of IR, ${ }^{1} \mathrm{H}$ - and ${ }^{13} \mathrm{C}-\mathrm{NMR}$ are summarized in Table 4.

Table 4, IR, ${ }^{1} \mathrm{H}$ and ${ }^{13} \mathrm{C}-\mathrm{NMR}$ Data of some common groups of QAS 4(a-d)-7(a-d)

\begin{tabular}{|c|c|c|c|c|c|c|c|}
\hline & \multicolumn{5}{|c|}{$\begin{array}{l}\text { Compositions and m.ps of } \\
\text { synthesized QAS 4(a-d)- } \\
7(\mathbf{a - d})\end{array}$} & \multirow[t]{2}{*}{ IR $v_{\max }, \mathrm{cm}^{-1}$} & \multirow[t]{2}{*}{$\begin{array}{c}{ }^{1} \mathrm{H}-\mathrm{NMR} \quad \delta,(\mathrm{ppm}) \\
{ }^{13} \mathrm{C}-\mathrm{NMR} \quad \delta,(\mathrm{ppm})\end{array}$} \\
\hline & $\mathrm{R}^{\mathrm{I}}$ & $\mathrm{R}^{2}$ & $\mathrm{R}_{3}$ & $\mathrm{R}^{4}$ & $\begin{array}{c}\mathrm{m} \cdot \mathrm{p}^{\circ} \\
\mathrm{C}\end{array}$ & & \\
\hline $4 a$ & $\mathrm{i}$ & $\mathrm{i}$ & iv & iv & 240 & $\begin{array}{l}3444 \\
\left(\mathbf{H}-\mathrm{N}^{+}\right)\end{array}$ & $\begin{array}{l}5.60\left(\underline{\mathbf{H}}_{-}-\mathrm{N}^{+}\right), 3.32\left(\underline{\mathbf{H}}_{2} \mathrm{C}-\mathrm{N}^{+}\right), 1.98\left(-\underline{\mathrm{C}}_{2}-\right) \\
46.94\left(\mathrm{H}_{2} \underline{\mathbf{C}}-\mathrm{N}^{+}\right), 26.34\left(-\underline{\mathbf{C}} \mathrm{H}_{2^{-}}\right) .\end{array}$ \\
\hline $4 b$ & $\mathrm{i}$ & $\mathrm{i}$ & iv & vii & & $\begin{array}{l}3445 \\
\left(\mathbf{H}-\mathrm{N}^{+}\right)\end{array}$ & $\begin{array}{l}5.53\left(\underline{\mathbf{H}}-\mathrm{N}^{+}\right), 3.30\left(\underline{\mathbf{H}}_{2} \mathrm{C}-\overline{\mathrm{N}}^{+}\right), 1.94\left(-\underline{\mathrm{CH}}_{2}-\right) \\
1.92(-\mathrm{C} \underline{\mathbf{H}}-\mathrm{cycl}) \\
47.26\left(\mathrm{H}_{2} \underline{\mathbf{C}}-\mathrm{N}^{+}\right), 37.92\left(-\underline{\mathrm{CH}}_{2^{-}}\right), 36.21(- \\
\underline{\mathbf{C}} \mathrm{H}-\mathrm{cycl}) .\end{array}$ \\
\hline $4 c$ & $\mathrm{i}$ & $\mathrm{i}$ & iv & viii & & $\begin{array}{l}3284(\mathbf{H}- \\
\left.\mathrm{N}^{+}\right) \\
1736(\mathbf{C}=\mathbf{O})\end{array}$ & $\begin{array}{l}5.37\left(\underline{\mathbf{H}}^{-\mathrm{N}^{+}}\right), 3.40\left(\underline{\mathbf{H}}_{2} \mathrm{C}-\mathrm{N}^{+}\right), 2.08\left(-\underline{\mathrm{C}}_{2}-\right), \\
6.40\left(-\underline{\mathrm{H}}_{2} \mathrm{CO}\right), 6.41(\mathrm{C} \underline{\mathbf{H}} \mathrm{O}-\mathrm{glu}) \\
46.95\left(\underline{\mathrm{H}}_{2} \underline{\mathbf{C}}-\mathrm{N}^{+}\right), 29.54\left(-\underline{\mathbf{C}} \mathrm{H}_{2^{-}}\right), \\
93.21(\underline{\mathbf{C H O}}-\mathrm{glu})\end{array}$ \\
\hline 4d & $\mathrm{i}$ & $\mathrm{i}$ & iv & ix & 127 & $\begin{array}{l}3419\left(\mathbf{H}-\mathrm{N}^{+}\right) \\
1633(\mathbf{C}=\mathbf{C})\end{array}$ & $\begin{array}{l}5.76\left(\underline{\mathbf{H}}-\mathrm{N}^{+}\right), 3.30\left(\underline{\mathbf{H}}_{2} \mathrm{C}-\mathrm{N}^{+}\right), 2.54\left(-\underline{\mathrm{C}}_{2}-\right), \\
1.48(-\mathrm{C} \underline{\mathbf{H}}-\mathrm{cycl}) . \\
50.17\left(\mathrm{H}_{2} \underline{\mathbf{C}}-\mathrm{N}^{+}\right), 29.50\left(-\underline{\mathrm{CH}}_{2^{-}}\right), 36.21(- \\
\text { CH-cycl }) .\end{array}$ \\
\hline $5 a$ & $\mathrm{i}$ & $\mathrm{i}$ & vi & iv & 96 & $\begin{array}{l}3444 \\
\left(\mathbf{H}-\mathrm{N}^{+}\right) \\
1640 \\
(\mathbf{C}=\mathbf{C} \text { Ar. })\end{array}$ & 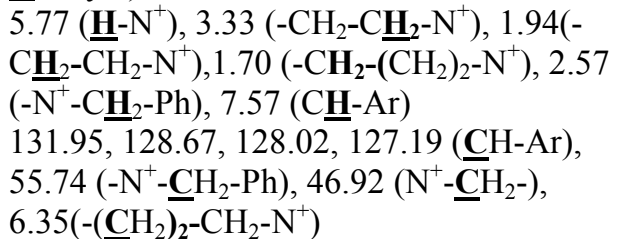 \\
\hline
\end{tabular}




\begin{tabular}{|c|c|c|c|c|c|c|c|}
\hline $5 b$ & $\mathrm{i}$ & $\overline{\mathrm{i}}$ & vi & vii & 92 & $\begin{array}{l}3361\left(\mathbf{H}-\mathbf{N}^{+}\right), \\
1643(\mathbf{C}=\mathbf{C} \\
\text { Ar.) }\end{array}$ & \\
\hline $5 \mathrm{c}$ & $\mathrm{i}$ & $\mathrm{i}$ & vi & $\begin{array}{l}\text { vii } \\
\text { i }\end{array}$ & & $\begin{array}{l}3423 \\
\left(\mathbf{H}-\mathrm{N}^{+}\right) \\
1725(\mathbf{C}=\mathbf{O})\end{array}$ &  \\
\hline $5 d$ & $\mathrm{i}$ & $\mathrm{i}$ & vi & ix & 122 & $\begin{array}{l}3400\left(\mathbf{H}-\mathrm{N}^{+}\right) \\
1644(\mathbf{C}=\mathbf{C} \\
\text { Ar. })\end{array}$ & $\begin{array}{l}5.37\left(\underline{\mathbf{H}}-\mathrm{N}^{+}\right), 3.55\left(\mathrm{~N}^{+}-\mathrm{C} \underline{\mathbf{H}}-\mathrm{cycl}\right), 2.00(- \\
\left.\mathrm{N}^{+}-\underline{\mathbf{H}}_{2}-\mathrm{Ph}\right), 8.41,7.82(\mathrm{C} \underline{\mathbf{H}}-\mathrm{Ar}) . \\
50.17\left(-\mathrm{N}^{+}-\underline{\mathbf{C H}}_{2}-\mathrm{Ph}\right), 140.79(\underline{\mathbf{C}}=\mathrm{C} \\
\text { aliphatic), } 130.78,128.30,128.62,128.50 \\
\text { (CH-Ar). }\end{array}$ \\
\hline $6 a$ & ii & ii & $\mathrm{v}$ & iv & 118 & $\begin{array}{l}1718(\mathbf{C}=\mathbf{O}), \\
1633(\mathbf{C}=\mathbf{C})\end{array}$ & $\begin{array}{l}4.07\left(\underline{\mathbf{H}}_{2} \mathrm{C}-\mathrm{N}^{+}\right), 3.67\left(-\underline{\mathbf{H}_{2}} \mathrm{C}-\mathrm{N}^{+}\right), 4.07(- \\
\left.\underline{\mathrm{H}}_{2} \mathrm{CO}\right), 6.06\left(\underline{\mathrm{C}}_{2} \mathrm{CO}\right), 4.59\left(\underline{\mathbf{H}}_{2} \mathrm{C}-\mathrm{N}^{+}\right) \\
62.21\left(\mathrm{H}_{2} \underline{\mathbf{C}}-\mathrm{N}^{+}\right), 51.79\left(-\mathrm{H}_{2} \underline{\mathbf{C}}-\mathrm{N}^{+}\right), \\
62.23\left(\mathrm{~N}^{+}-\underline{\mathbf{C}} \mathrm{H}_{2} \mathrm{CH}_{2}-\mathrm{O}\right), 24.69\left(-\underline{\mathbf{C}}{ }_{2} \mathrm{CO}\right) \\
166.30\left(-\overline{\mathrm{CH}}_{2} \mathbf{C O}\right) .\end{array}$ \\
\hline $6 b$ & ii & ii & $\mathrm{v}$ & vii & & $\begin{array}{l}1729(\mathbf{C}=\mathbf{O}), \\
1633(\mathbf{C}=\mathbf{C})\end{array}$ & $\begin{array}{l}3.33\left(-\mathrm{N}^{+}-\mathrm{C} \underline{\mathbf{H}}_{3}\right) ; 4.18\left(\mathrm{~N}^{+}-\underline{\mathbf{H}}_{2} \mathrm{C}-\right), 4.61(- \\
\left.\mathrm{C} \underline{\mathbf{H}}_{2}-\mathrm{O}-\mathrm{CO}\right), 3.08(\underline{\mathbf{H}}-\mathrm{C}-\text { cyclohexyl }), 4.62 \\
\left(\underline{\mathbf{H}}^{-} \mathrm{C}=\mathrm{C}\right), 1.58\left(\mathrm{C} \underline{\mathbf{H}}_{3}-\mathrm{C}=\mathrm{C}\right) . \\
61.26\left(-\mathrm{N}^{+}-\underline{\mathbf{C}} \mathrm{H}_{3}\right) ; 53.96\left(-\mathrm{N}^{+}-\underline{\mathbf{C H}}_{2}\right) ; 63.00 \\
\left(-\underline{\mathbf{C}} \mathrm{H}_{2}-\mathrm{O}-\mathrm{CO}\right) ; 56.56(\mathrm{H}-\underline{\mathbf{C}}-\mathrm{cyclohexyl}) ; \\
134.51\left(\mathrm{CH}_{2}-\mathrm{C}=\underline{\mathbf{C H}}_{2}\right) .\end{array}$ \\
\hline $6 c$ & ii & ii & $\mathrm{V}$ & viii & & $\begin{array}{l}1724(\mathbf{C}=\mathbf{O}), \\
1645(\mathbf{C}=\mathbf{C})\end{array}$ & $\begin{array}{l}4.10\left(-\mathrm{N}^{+}-\mathrm{C} \underline{\mathbf{H}}_{3}\right) ; 4.30\left(\mathrm{~N}^{+}-\underline{\mathbf{H}}_{2} \mathrm{C}-\right), 4.58(- \\
\left.\mathrm{CH}_{2}-\mathrm{O}-\mathrm{CO}\right), 4.31\left(\mathrm{~N}^{+}-\mathrm{C} \underline{\mathbf{H}}-\mathrm{O}-\text { gluco }\right), 4.72 \\
\left(\underline{\mathrm{C}}_{3}-\mathrm{CO}\right) .\end{array}$ \\
\hline $6 d$ & ii & ii & $\mathrm{v}$ & ix & 137 & $\begin{array}{l}1728(\mathbf{C}=\mathbf{O}), \\
1633\left(\mathbf{C}=\mathbf{C} \mathbf{H}_{2}\right)\end{array}$ & $\begin{array}{l}3.33\left(-\mathrm{N}^{+}-\mathrm{C} \underline{H}_{3}\right) ; 5.34\left(\mathrm{~N}^{+}-\underline{H}_{2} \mathrm{C}-\right), 4.64(- \\
\left.\mathrm{CH}_{2}-\mathrm{O}-\mathrm{CO}\right), 3.41(\underline{\mathbf{H}}-\mathrm{C}-\text { cyclohexyl }), 5.67 \\
(\underline{\mathbf{H}}-\mathrm{C}=\mathrm{C}), 76.95\left(-\mathrm{N}^{+}-\underline{\mathbf{C}} \mathrm{H}_{3}\right) ; 59.35\left(\mathrm{~N}^{+}-\right. \\
\left.\mathrm{H}_{2} \underline{\mathbf{C}}-\right), 50.48\left(-\mathrm{CH}_{2} \underline{\mathbf{C}}=\mathrm{O}\right), 135.58\left(\mathrm{CH}_{3^{-}}\right. \\
\underline{\mathbf{C}}=\mathrm{C}), 141.12(-\underline{\mathbf{C}}=\mathrm{H}=), 166.88(\underline{\mathbf{C O}}- \\
\left.\overline{\mathrm{C}} \mathrm{H}_{2}\right) .\end{array}$ \\
\hline $7 a$ & iii & iii & $\mathrm{v}$ & iv & 117 & $\begin{array}{l}1728(\mathbf{C}=\mathbf{O}) \\
1636 \\
\left(\mathbf{C}=\mathbf{C} \mathbf{H}_{2}\right)\end{array}$ & $\begin{array}{l}3.57\left(\underline{\mathbf{H}}_{2} \mathrm{C}-\mathrm{N}^{+}\right), 3.87\left(-\mathrm{C}_{2}-\mathrm{CH}_{2}-\mathrm{N}^{+}\right), 4.57 \\
\left(-\mathrm{CH}_{2}-\underline{\mathbf{C}}_{2}-\mathrm{N}^{+}\right), 6.04(\underline{\mathbf{H}}-\mathrm{C}=\mathrm{C}) . \\
57.86\left(\mathrm{H}_{2} \mathbf{C}-\mathrm{N}^{+}\right), 58.74\left(\mathrm{~N}^{+}-{ }_{\mathbf{C}} H_{2} \mathrm{CH}_{2}-\mathrm{O}\right), \\
24.02\left(\mathrm{~N}^{+}-\mathrm{CH}_{2} \underline{\mathbf{C}}_{2}-\mathrm{O}\right), 166.35\left(-\mathrm{CH}_{2} \underline{\mathbf{C O}}\right), \\
135.1\left(\mathrm{C}=\underline{\mathbf{C}}-\mathrm{CH}_{2}\right) .\end{array}$ \\
\hline $7 b$ & iii & iii & $\mathrm{v}$ & vii & 108 & $\begin{array}{l}1720(\mathbf{C}=\mathbf{O}) \\
1635 \\
\left(\mathbf{C}=\mathbf{C} \mathbf{H}_{2}\right)\end{array}$ & \\
\hline $7 c$ & iii & iii & $\mathrm{v}$ & viii & & $\begin{array}{l}1728(\mathbf{C}=\mathbf{O}) \\
1636 \\
\left.\mathbf{C}=\mathbf{C H}_{2}\right)\end{array}$ & $\begin{array}{l}3.33\left(\underline{\mathbf{H}}_{2} \mathrm{C}-\mathrm{N}^{+}\right), 3.94\left(-\mathbf{C H}_{2}-\mathbf{C H}_{2}-\mathrm{N}^{+}\right), 4.53 \\
\left(-\mathrm{C}_{2}-{ }^{-} \mathrm{CH}_{2}-\mathrm{N}^{+}\right), 4.74\left(\mathrm{~N}^{+}-\mathrm{CH}_{2} \mathrm{C} \underline{\mathbf{H}}_{2}-\mathrm{O}\right), \\
4.20,4.06(\underline{\mathbf{H}}-\mathrm{C}-\mathrm{O}-\mathrm{Ac}) .\end{array}$ \\
\hline 7d & iii & iii & $\mathrm{V}$ & ix & 148 & $\begin{array}{l}1727(\mathbf{C}=\mathbf{O}), \\
1647\left(\mathbf{C}=\mathbf{C H}_{2}\right)\end{array}$ & \\
\hline
\end{tabular}




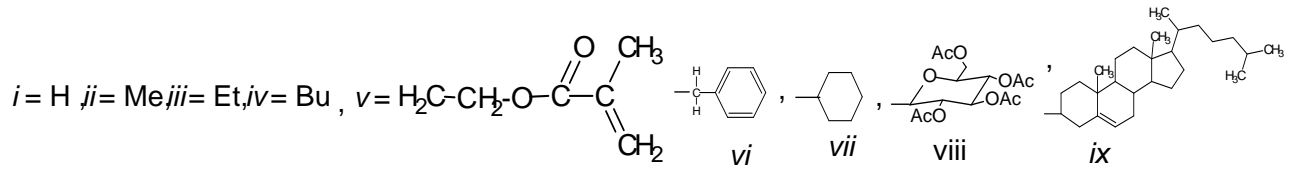

Characterization of Groups-C \&D=[alkyl-DMAEMAB] 6(a-d) \& [alkyl-DEAEMAB]7(a-d)

Groups $\mathrm{C}$ and $\mathrm{D}$ have almost similar structural formulas represented by $N, N$-dialkyl amino ethyl-2- methyl acrylate fragments, for Group- $\mathrm{C}$ and for $\mathrm{R}=\mathrm{H}$, Group-D, $\mathrm{R}=-\mathrm{CH}_{3}$ :<smiles>[R]C[N+]([R])([R4])CCOC(=O)C(=C)C</smiles>

Figure 2. The basic structural formulas for Groups C and D

Thus all compounds 6(a-d)-7(a-d) showed IR absorption between 1728-1718 $\mathrm{cm}^{-1}(\mathrm{CO})$, 1647-1633 $\mathrm{cm}^{-1}(\mathrm{C}=\mathrm{C})$. Also compounds exhibited signals between 4.10-3.33 ppm corresponding to $\mathrm{N}^{+}-\mathrm{CH}_{2}-\mathrm{R}, 4.74-4.07$ due to $-\mathrm{N}^{+}-\mathrm{CH}_{2}-\mathrm{CH}_{2}-\mathrm{O}-$, , 124-127 ppm and 166-167 ppm related to $\mathrm{C}=\mathrm{CH}_{2}$ and $\mathrm{CO}$ respectively ${ }^{25}$.

\section{Antibacterial activity}

The sixteenth QAB 4(a-d)-7(a-d) were screened for their activity against Gram-positive Staphylococcus aureus and Gram-negative bacteria, Pseudomonas aeruginosa, Pseudomonasfluorescens and Shigella. The antibacterial activities of the tested compounds were evaluated using the paper disk diffusion method. DMSO which is known as bacterial static in the above mentioned concentration was used as negative control and standard disks (Mast Diagnostics, UK), saturated with known antibiotic Gentamycinand Amoxicillinas positive control were applied. After incubation at $37{ }^{\circ} \mathrm{C}$ for $24 \mathrm{~h}$, the zone of inhibition of growth around each disk was measured in millimeters and zone diameters were interpreted in accordance with CLSI and NCCLS (for Campylobacter spp.) guidelines (CLSI, 2006; NCCLS, 2003, 2005) $)^{26-28}$. The experiments were performed in duplicates and the average results are summarized in Table 5.

Table 5. Antibacterial activity of the synthesized compounds

\begin{tabular}{|c|c|c|c|c|}
\hline \multirow[b]{2}{*}{ Compounds } & \multicolumn{4}{|c|}{ Inhibition Zone, $\mathrm{mm}$} \\
\hline & Shigella & $\begin{array}{l}\text { Pseudomonas } \\
\text { fluorescens }\end{array}$ & $\begin{array}{c}\text { Pseudomonas } \\
\text { aeruginosa }\end{array}$ & $\begin{array}{c}\text { Staphylococcus } \\
\text { aureus }\end{array}$ \\
\hline $4 a$ & 10.00 & 18.00 & 00.00 & 25.00 \\
\hline $4 b$ & 08.00 & 18.00 & 00.00 & 25.00 \\
\hline $4 c$ & 15.00 & 00.00 & 00.00 & 36.00 \\
\hline $4 d$ & 00.00 & 00.00 & 00.00 & 10.00 \\
\hline $5 a$ & 15.00 & 15.00 & 20.00 & 18.00 \\
\hline $5 b$ & 00.00 & 15.00 & 00.00 & 30.00 \\
\hline $5 c$ & 00.00 & 10.00 & 00.00 & 20.00 \\
\hline $5 d$ & 00.00 & 00.00 & 00.00 & 20.00 \\
\hline $6 a$ & 10.00 & 10.00 & 00.00 & 25.00 \\
\hline $6 b$ & 15.00 & 17.00 & 30.00 & 15.00 \\
\hline $6 c$ & 13.00 & 13.00 & 20.00 & 15.00 \\
\hline
\end{tabular}




\begin{tabular}{ccccc}
\hline $\boldsymbol{6 d}$ & 00.00 & 00.00 & 00.00 & 16.00 \\
$\mathbf{7} \boldsymbol{a}$ & 15.00 & 13.00 & 00.00 & 20.00 \\
$\mathbf{7 b}$ & 00.00 & 12.00 & 30.00 & 08.00 \\
$\mathbf{7} \boldsymbol{c}$ & 20.00 & 10.00 & 00.00 & 15.00 \\
$\mathbf{7 d}$ & 00.00 & 00.00 & 00.00 & 10.00 \\
DMSO & 00.00 & 00.00 & 00.00 & 00.00 \\
Gentamicine & 30.00 & 30.00 & 45.00 & 25.00 \\
Amoxicilline & 25.00 & 25.00 & 40.00 & 45.00 \\
\hline
\end{tabular}

All QAB 4(a-d)-7(a-d) have affected gram-positive Staphylococcus aureusin active range compatible to known antibiotic Gentamicin and moderately active comparable to Amoxicillin. Compound glucosidyl-ABB (4c) showed the highest activity against $S$. aureus followed by compound cyclohexyl -ABzB (5b).

All QAB 4(a-d)-7(a-d) showed less activity against gram-negative bacteria under study. Shigella exhibited about $44 \%$ resistance to all synthesized QAB while Pseudomonas fluorescens showed about $31 \%$ resistance. Pseudomonas aeruginosawas resistant to about $76 \%$ of the synthesized $\mathrm{QAB}$ except four compounds $\mathbf{5 a}, \mathbf{6 b}, \mathbf{6 c}$ and $\mathbf{7 b}$ which showed moderate to weak activity.

Microorganisms not affected by compounds tested, no minimum inhibition concentration (MIC) test were conducted. Those which showed activity, MIC was done by dilution with DMSO to $5.0 \mu \mathrm{g} \mathrm{mL}^{-1}$ but they show almost no significant activity.

\section{Conclusion}

The work in this study is beneficial and valuable to those who are interested in studying antibacterial activity for new QAC and particularly with resin composites such as the acrylate QABs.

\section{Acknowledgment}

Thanks are due to Mrs.F.Z. Benmiloudi, M.Sc, Miss. F. Taieb Brahimi, M.Sc. and Miss. I. Taghaneth, M.Sc., of the Laboratory of Bioactive Organic Chemistry, Department of Chemistry, University of Sciences and Technology of Oranfor performing the biological test.

\section{References}

1. Bhat S, Leikin-Gobbi D, Konikoff F M and Maitra U, Biochimica et Biophysica Acta (BBA) - General Subjects, 2006, 1760(10), 1489-1496.

2. Zhang Z H, Janout V, Renner J L, Urgami M and Regan S L, Bioconjugate Chem., 2000, 11(3), 397-400; DOI:10.1021/bc990151v.

3. Fears R, Brown R, Ferres H, Grenier F and Tyrrell A W R, Biochem Pharmacol., 1990, 40(9), 2029-2037; DOI:10.1016/0006-2952(90)90233-B.

4. Fini A, Roda A, Billini A M, Mencini E and Quarneri M, J Pharm Sci., 1990, 79(7), 603-605; DOI:10.1002/jps.2600790712.

5. Kwan K H, Higuchi W I, Molokhia A M and Hofmann A F, J Pharm Sci., 1977, 66(8), 1105-1108; DOI:10.1002/jps.2600660813.

6. Walker S, Sofia M J and Axelrod H R, Adv Drug Delivery Rev., 1988, 30(1-3), 61-71; DOI:10.1016/S0169-409X(97)00107-5.

7. Bandyopadhyaya P, Lanout V, Zhang L H, Sawko J A and Regan S L, J Am Chem Soc., 2000, 122(51), 12888-12889; DOI:10.1021/ja0056466.

8. Brycki B, Kowalczyk I and Kozirog A, Molecules, 2011, 16(1), 319-335; DOI:10.3390/molecules16010319. 
9. Ohta Y, Kondo Y, Kawada K, Ternaka T and Yoshino N, J Oleo Sci., 2008, 8, 445-452.

10. Xiao Y H, Chen J H, Fang M, Xing X D, Wang H, Wang Y J and Li F, J Oral Sci., 2008, 50(3), 323-327.

11. Makhloufia M, Benaissa T, Derdour A and Henni D E, J Chem., 2010, 7(S1), 561-566.

12. He J, Soderling E, Osterblad M, Vallittu P K and Lassila L V J, Molecules, 2011, 16(11), 9755-9763; DOI:10.3390/molecules16119755.

13. Weng Y, Guo X, Chong V J, Howard L, Gregory R L and Xie D, J Biomed Sci Eng., 2011, 4, 147-157.

14. Dega-Szafron Z, Dulewicz E and Brycki B, Arkivoc, 2007, (vi), 90-102.

15. de Britto D, Goy R C, Filho S P C and Assis O B G, Int J Carbohy Chem., 2011, Article ID 312539, 12 pages; DOI:10.1155/2011/312539

16. Richter F L and Cords B R, Disinfiction, Sterilization and Preservation, Block S S, Editor, $5^{\text {th }}$ Ed., Lippincott Williams \& Wilkins, Philiadelphia, 9106, USA. 2001.

17. Zhu P and Sun G, J Appl Polym Sci., 2004, 93(3), 1037-1041;

DOI:10.1002/app.20563.

18. Lang G, Wendel H and Birkel S, in “ Chitin Handbook”, Muzzarelli R A A and Peter M G, Eds., European Sciety Press, Grottanmmare, Italy, 1997, 384-389.

19. Ringdahl B, Roch M and Jenden D J, J Med Chem., 1988, 31(1), 160-164; DOI:10.1021/jm00396a024.

20. Mac Farlane D R, Forsyth S A, Golding J and Deacon G B, Green Chem., 2002, 4, 444-448; DOI:10.1039/B205641K.

21. Tigelaar D M, Meador M A B and Bennett W R, Macromolecules, 2007, 40(12), 4159-4164; DOI:10.1021/ma062804q.

22. Furniss B S, Hannford A J, Smith P W G and Tatchell A R, Vogel's Text Book of Practical Organic Chemistry, 5/e, Longman Scientific \& Technical, New York, 1989, 647.

23. Gao M, Wang M, Miller K D, Sledge G W, Hutchins G D and Zheng Q H, Steroids, 2010, 75, 715-720.

24. Pretsch E, Buhlmann P and Affolter C, Structure Determination of Organic Compounds - tables of spectral data, Springer-Verlage Berlin Heidelberg, 2000, 254.

25. Pretsch E, Buhlmann P and Affolter C, Structure Determination of Organic Compounds-tables of spectral data, Springer-Verlage Berlin Heidelberg, 2009, p 161,71 .

26. Dallal M M S, Doyle M P, Rezadehbashi M, Dabiri H, Sanaei M, Modarresi S, Bakhtiari R, Sharifiy K, Taremi M, Zali M R and Sharifi-Yazdi M K, Food Control, 2010, 21(4), 388-392; DOI:10.1016/j.foodcont.2009.06.001.

27. Clinical and Laboratory Standards Institute, Performance Standards for AntimicrobialSusceptibily Testing; Sixteenth International Supplement. 2006, 26, 11-35.

28. National Committee for Clinical and Laboratory Standards Villanova, PA, Methods for Dilution Antimicrobial Susceptibility Tests for Bacteria that Grow Aerobically, 4/e, 1997. 